\title{
AN EXPERIMENT-DRIVEN MASS-PERSONALISATION MODEL: APPLICATION TO SAXOPHONE MOUTHPIECE PRODUCTION
}

\author{
M. Ozdemir $\bowtie$ and G. Cascini \\ Politecnico di Milano, Italy \\ $\triangle$ mehmet.ozdemir@polimi.it
}

\begin{abstract}
Mass-personalization (MP) presents an opportunity to meet diversifying customer needs in consumer products market with a near mass-production efficiency. Traditional product development methodologies fall short to guide design for MP and a dedicated systematic methodology is essential. The proposed approach bases on a dynamic product template that automatically adapts with user input and produces a reliable output. This paper presents the workflow towards mass-personalization of saxophone mouthpieces with focus on design automation.
\end{abstract}

Keywords: mass personalization, additive manufacturing, design methods, co-design

\section{Introduction}

In the past years, Mass-Customization (MC) has been employed to match the needs of specific customers at low cost (Wind and Rangaswamy, 2001). Nevertheless, many trials have failed due to both not being able to match user expectations and the creation of unnecessary cost and complexity (Christy, 1993; Zipkin, 2001). To match the customer needs (CNs) better, a higher degree of customization is possible to be applied by tailored products at elevated costs. At this point, emerging Additive Manufacturing (AM) technologies present an opportunity to provide personalization at no additional manufacturing cost, as these technologies advance towards manufacturing of consumer products (CPs). Although studies demonstrate that consumers are more engaged to personalized products and willing to pay more, they are also not willing to pay much more compared to mass-produced products (Abdul Kudus et al., 2016; Campbell et al., 2014). This brings forward the concept of mass-personalization (MP), where dedicated time and labour to each customer is aimed to be eliminated by providing automation to the personalization process with user-modifiable product templates. MP is anticipated to be a major driver for the next transformation of the global economy (Tseng et al., 2010). This relatively new concept raises several questions about the product development process of MP products and the role of the designer in the process. The future role of the designer in consumer products is envisioned to be a facilitator of CNs by designing unfinished products to be finalized by the users while ensuring safe and reliable output (Sinclair and Campbell, 2014; Siu, 2003). The arising concept for such a scenario is the development of a product template by the designer, where certain design features or parameters are defined as a range. With a given form of user involvement, this then gives each user the ability to individualize the product in predetermined design space (Kuksa and Fisher, 2017). The main question arising from this point, addressed by this research, is how a user-modifiable product template or seed design should be developed within AM context. The lack of methods and tools in this domain has been 
pointed out by previous research (Campbell et al., 2014; Kuksa and Fisher, 2017). There are few successful applications of personalization with AM, mainly in the medical field, such as teeth aligners or hearing aids. It is possible to broaden these applications to a broader range of consumer products by defining a systematic design methodology.

The long-term aim of this research is to establish a design methodology for MP that sheds light on how to develop products in this new paradigm. The expected outcome is a set of guidelines and tools to support designers in developing product templates that are personalisable in an automated manner. This paper aims to explore such an original product development process on a saxophone mouthpiece personalization case. The following sections describe the seed design development process for a saxophone mouthpiece and the preliminary evidence of the validity of the proposed approach. More rigorous validation of the design methodology is in progress through a user study on saxophone players.

\section{Background and related work}

Product development methodologies for mass-production have been well-established long ago (Pahl et al., 2007; Ulrich and Eppinger, 2012). In the traditional product development for mass production, the process is done based on identified user requirements and a design solution is generated. These methodologies are also adaptable to MC cases, where only certain product configurations are provided. In MP on the other hand, since the aim is to respond to differentiating user needs or desires, a dynamic model is needed where the product is tailored within a predefined design space. A generic framework has been presented for MP from product planning to supply chain (Tseng et al., 2010). Another similar work has been done on both product and service design personalisation with a lifecycle perspective (Kaneko and Kishita, 2017). Ariadi and Rennie (2008) defined a design template concept for personalisation and located it in the product development process. A further addition has been done in a framework of MP for service design, providing a co-creation model (Hsiao et al., 2015). The previous research on MP has mainly focused on providing workflows from a broad perspective. While there is a consensus on using some form of product template to automate the personalisation process, there is also a lack of knowledge about how such product template should be developed. Since this new product development paradigm is mostly viable by AM, design considerations in that domain are also relevant. Design for AM (DfAM) methodologies have been studied widely in the last years, mainly focusing on novel concept generation and application of design rules/guidelines (Laverne et al., 2015; Rias et al., 2016; Zhu et al., 2017). Spallek et al. (2016) demonstrated an overall process integrating personalisation with AM, while not in a mass way.

To automate the design process, Axiomatic Design (AD) and Design Structure Matrix (DSM) principles set convenient examples. AD provides an established method for translating CNs into functional requirements (FRs) and design variables (DVs) (Salonitis, 2016). While DSM provides a clear representation of the dependencies and provides solutions to reach a design solution. Both methods are aimed at a single case to reach a specific solution. However, these methods could be adopted for a dynamic multiple solution case of MP. Since each FR and DV would be a range in a seed design, rather than a single value as in traditional product development, the dynamic relationships need to be explained. These dynamic relationships create some constraints on possible design configurations, along with the design and manufacturing constraints. A better understanding of the limitations they enforce on each other is necessary to treat this complex problem.

\section{Saxophone mouthpieces}

A saxophone mouthpiece (Figure 1) is the interface between the player and the instrument, where the sound is produced by the vibration of a reed attached to the mouthpiece. Therefore, it is safe to say that in terms of acoustics, the mouthpiece is the most essential part of the saxophone, by not only producing but also shaping the sound. Besides, as being the interface, playability of the instrument is primarily defined by the mouthpiece. Consequently, there is an everlasting quest of finding the most suitable mouthpiece among saxophone players. Besides having a certain demand for personalization, saxophone mouthpiece is a product with a rather simple design and high value. This makes it a very suitable candidate for personalization and on-demand production by AM. 


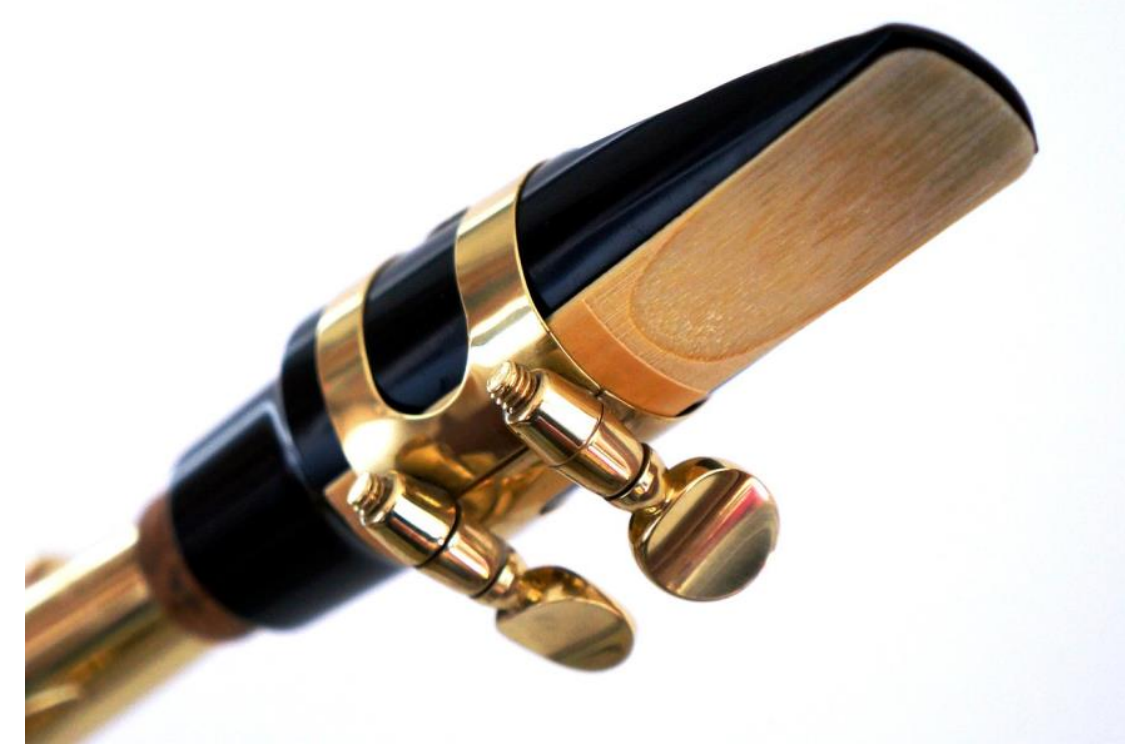

Figure 1. Alto saxophone mouthpiece

The saxophone mouthpiece design is still to this date an artisanal work based on experience, and it is well-known that even small changes in the design differ them significantly (Teal, 1963). However, since the design has been based on intuition and often with changing multiple parameters, the relative effect of each parameter is not apparent and there are several misconceptions (Hasbrook, 1996). Features of the mouthpiece are considered to be strictly related to the characteristics of the airflow through the chamber, and there have been few attempts to clarify the working principle of the mouthpiece (Lorenzoni et al., 2013). However, since the focus of this work is on personalization, the approach is on rather identifying the effects of well-established design parameters and building a modifiable product template based on this.

Saxophone mouthpieces most commonly made of hard rubber or various metals and rarely found in wood, porcelain or glass. Although the manufacturing is partially done by subtractive machining today, finishing is still done by manual labour in most of the cases. This is the major driver of the high cost of mouthpieces. Regarding this, AM could provide substantial ease of manufacturing and reduction of costs.

\section{Development process}

\subsection{Proposed framework}

The proposed methodology integrates DfAM into the conception of a general product development scheme, while instead of aiming a definite product as an output, a user-modifiable product template, a seed design, which has pre-defined and assured ranges of variables. The product development process is then followed by a consumer co-creation activity, where the consumer individualizes the product within the pre-defined boundaries. The aim is to automate the process of obtaining personalized products, defining the seed design once and then the co-creation is repeated by each customer. Consequently, it is expected to eliminate the need for dedicated labour to each customer while adding value to AM products by personalization.

To automate the design process, the proposed methodology investigates the relationships between the domains of FRs by users and DVs. The general framework proposed is outlined in Figure 2. Instead of a single design solution, a seed design with a range of variables is developed. The seed design then finalized with the contribution of the user through a co-creation process. Since the design choices specified by the user possibly affect the boundaries of the design space for following requirements, a dynamic interaction between the seed design and the user is necessary. This implies that by each design choice made, consequences on further choices becomes evident. Once all the design choices are made, the personalized final design is generated. 


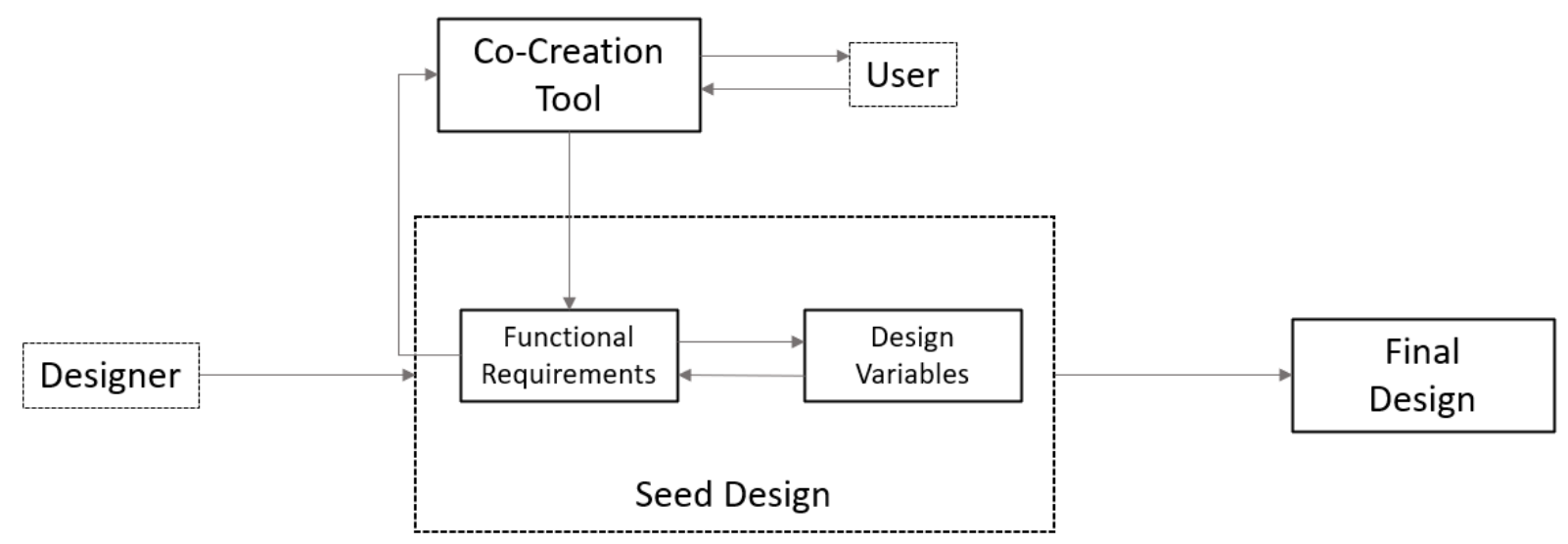

Figure 2. Proposed framework

This model entails identification of personalizing requirements and mapping these onto relevant design variables. The interdependencies of the variables and consequently, of the requirements are needed to be examined to generate a valid design solution. To achieve a dynamic design space, the ranges of the variables are required to be predefined in a conditional way, which consequently defines the ranges of functional requirements. To achieve a final design, variables are iterated following each input from the user. In the following section, these concepts are explained further.

\subsection{Seed design development}

\subsubsection{Functional requirements}

In the case of a saxophone mouthpiece, possible aspects of personalization can be categorized as sound characteristics and playing conditions. Most of the aspects related to sound are based on sound perception and psychoacoustics, which are very subjective and difficult to quantify (Carron et al., 2017). Since in the scope of this study quantified relations are needed, two measurable sound characteristics are identified; tone colour and volume of the sound. Tone colour is measured with the spectral centroid of the sound, which can be also defined as the brightness. Volume implies the loudness of the instrument and measured by the intensity of the sound waves. In terms of playing conditions, resistance and flexibility are identified as quantifiable aspects. Resistance is the ease of blowing the mouthpiece and measured by the minimum blowing pressure to produce sound. Flexibility implies the range of possible pitch alteration on the mouthpiece, and it is measured by the highest and lowest pitch produced.

\subsubsection{Design variables}

The design features affecting the performance of the mouthpiece are the ones at the interface between reed and mouthpiece and the ones defining the inner geometry (Figure 3). These wellestablished features have been identified by the artisans and manufacturers. These may be argued to be redefined to eliminate the dependencies and have a one-to-one mapping with requirements. However, this would be a very challenging, if not impossible, task. Since, as stated before, the mechanism behind is not clear and previous attempts to explore have not been conclusive yet. Due to the experimental setup used, it was not possible to observe the effects of changes in tip and side rail widths, as these are related to the articulation of the player. Similarly, beak size changes the oral cavity of the player and since this is fixed in the experiment, it is left out as well. Changes in the table and window lengths did not appear to have significant effects in measurements, and hence they are excluded from the final model. In conclusion, the design variables used can be listed as tip opening (TO), lay length (LH), chamber shape (CP), chamber size (CS), throat shape (TP), throat size (TS) and baffle height (BH). 

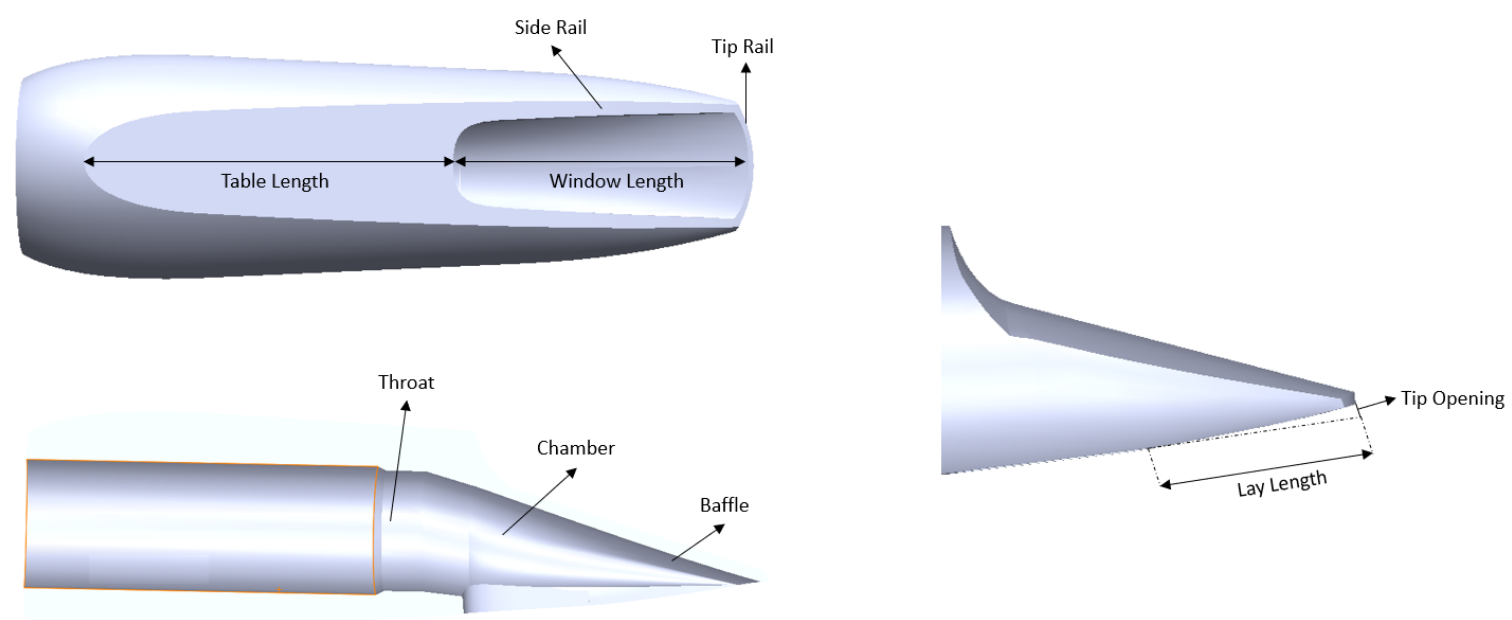

Figure 3. Design variables of the mouthpiece

\subsubsection{Operable ranges}

While defining the design space, three levels of restrictions can be considered. The initial and the broadest would be considering the possible interval of each design variable separately. One way to identify this is to examine the existing products in the market. A hundred Alto Saxophone mouthpieces were analysed and the variability of their characterizing dimensions recorded. For instance, Figure 4 shows the relationship between the tip opening and the lay length. In this case, the interval of the tip opening would be 1.4-2.6 mm, and the interval of lay length would be $17-25 \mathrm{~mm}$. It should also be noted that, as it is evident in the figure, there is also unexplored design space. Therefore, besides tailoring the product, this practice may lead to exploring different possibilities. One level further narrowing down on the design space shall be done considering manufacturing constraints and functional restrictions. In the mouthpiece case, there are no significant manufacturing constraints as the design is relatively simple, and the changes are on a small scale. Nevertheless, there are some functional restrictions which are related to the playability. An example of this might be a case of very high baffle and very small tip opening. Since this case results in a very small cross-sectional area for airflow, playability of the mouthpiece would be very poor. The last level of restrictions on the design space is related to the mutual dependencies between the variables and their consequent possible ranges. Each design choice defines a temporary design space until the next choice is made. For instance, when a choice is made on tone colour, due to having common variables, this enforces a narrower interval for the volume.

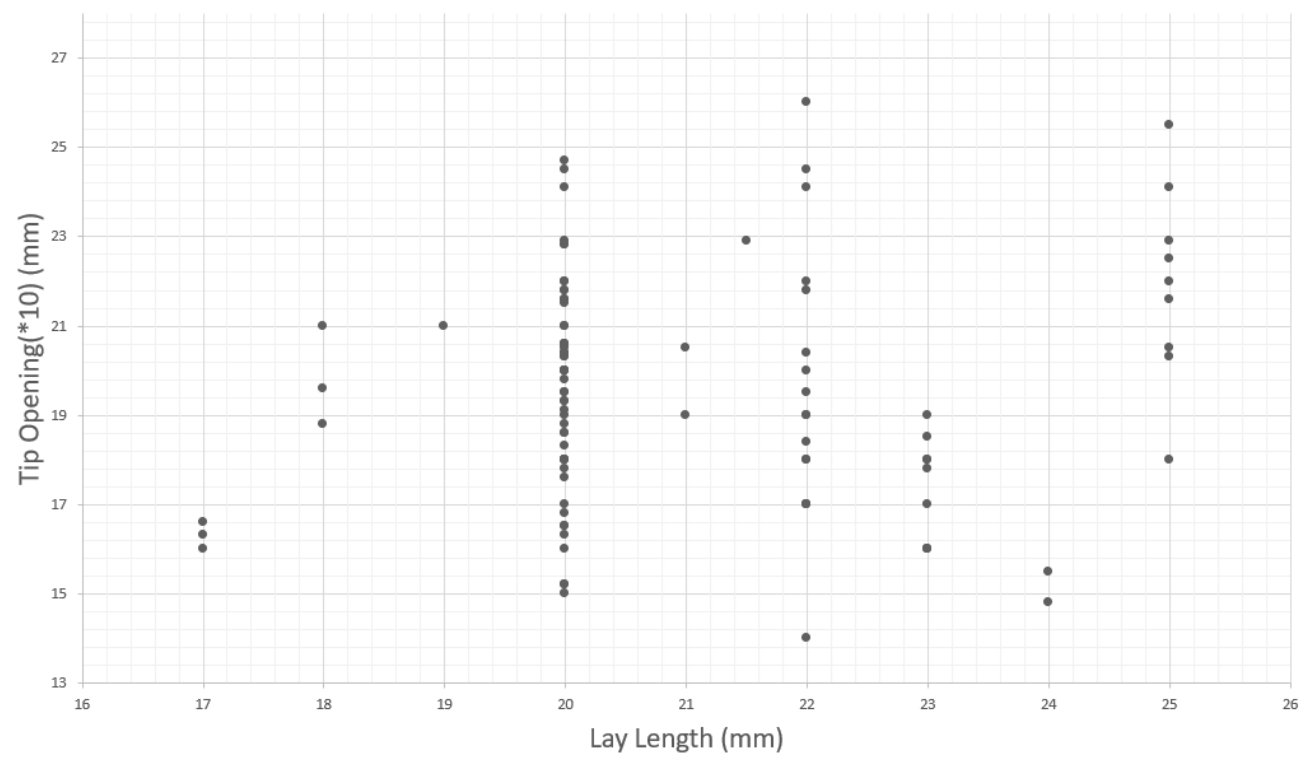

Figure 4. Tip opening vs. lay length of 100 alto saxophone mouthpieces in the market 


\subsubsection{Mapping and design solution}

Final mapping of FRs onto DVs is demonstrated in Figure 5. As it is seen in the mapping, all the variables affect multiple requirements. Therefore, in Axiomatic Design terms, it turns out to be a highly coupled design task. It should be noted here that these relationships are indeed quantified by experimental acquisitions through a DOE, as detailed in section 4.3. For the sake of the simplicity of the argument of this paper, these are left out for the follow-up work.

\begin{tabular}{|c|c|c|c|c|c|c|c|}
\hline Tone Color & & $x$ & & $x$ & $x$ & $x$ & \\
\hline Volume & $x$ & $x$ & & $x$ & $x$ & $x$ & $x$ \\
\hline Flexibility & $x$ & & & & & $x$ & $x$ \\
\hline Resistance & $x$ & $x$ & $x$ & & & $x$ & $x$ \\
\hline & 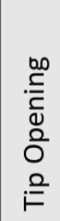 & 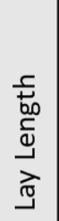 & 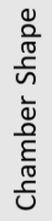 & 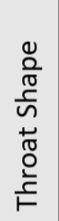 & 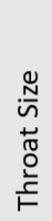 & 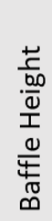 & 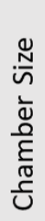 \\
\hline
\end{tabular}

Figure 5. Mapping of FRs onto DVs

One of the most crucial points in reaching a design solution is engaging the user in the process by providing feedback on the consequences of each design choice. In this case, there are four options presented to the user. Since the importance of each requirement is also a personal choice, the user should be aware that there are trade-offs and priority should be given to most desired aspects.

To achieve a personalized final design, an iterative approach is preferred. Since there are multiple variables affecting the requirements, the user request can be satisfied with different variables, and hence there are possibly multiple solutions. Nonetheless, the aim is to find the solution for each FR which gives more freedom on others. Initially, the seed design is pre-set to the mean values of each DV. The core idea is to change minimum DVs at each step to keep the largest interval for the remaining FRs. Therefore, to satisfy an FR, initially, the DV with the highest influence is changed. When the first DV reaches an extreme end of its variability range, the second-highest influencing DV is changed. The stepwise iteration of DVs goes on this way from the highest to the lowest influencing until the FR is satisfied.

\subsection{Prototyping and experimentation}

In order to obtain the overall dimensions and design attributes, the process begins with reverse modelling an existing mouthpiece. For this purpose, a traditional and well-established design, a Yanagisawa ebonite alto saxophone mouthpiece (no: 6), has been used. For 3D scanning of the object, a Nikon VIVID 9i laser scanner was used. The scanner has $0.03 \mathrm{~mm}$ Z-Depth and $0.145 \mathrm{~mm} \mathrm{X \& Y}$ resolution, which is just adequate for this application. The output point cloud of the scan has been processed via Polyworks software and converted into a mesh model. The mesh model then processed through Scanto3D module of Solidworks and a parametric model of the Yanagisawa mouthpiece has been obtained. Based on the obtained parametric model, a mouthpiece template has been developed. Since the design template needs to be dynamic and adaptable to user requirements, Grasshopper module of Rhinoceros has been used.

Several 3D printing technologies have been tested and compared for manufacturing the mouthpieces. Due to the high accuracy, resolution and repeatability of the results, stereolithography was chosen as the manufacturing method. Since the design parameters considered to have a sensitivity of $0.1 \mathrm{~mm}$, this is a limiting factor for certain technologies. E.g. mouthpieces manufactured by FDM technology had an inconsistency of up to $1 \mathrm{~mm}$ in repeated prints. Therefore, for the 3D printing, a Formlabs Form 2 printer has been used (Figure 6). Post-processing of the prints has been done respectively in Form wash and Form cure modules. Standard translucent resin has been used for the prints. The machine is capable of printing 16 mouthpieces at a time, which lasts about 19 hours at $0.1 \mathrm{~mm}$ resolution. Post-processing steps took about another hour in total. 


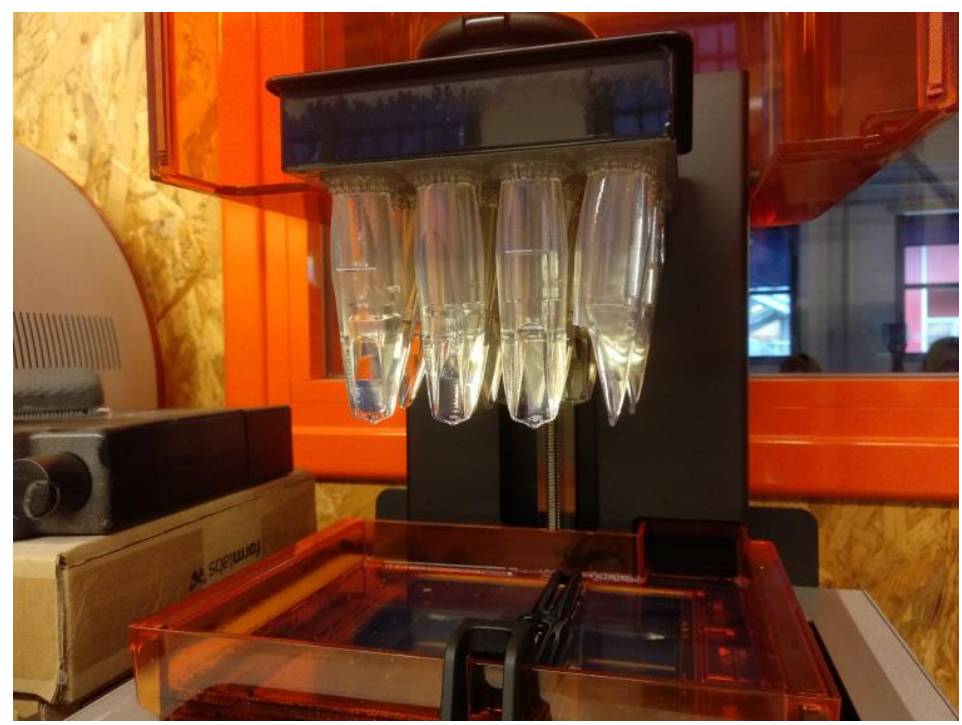

Figure 6. Prototyping of the mouthpieces

As stated before, the effect changes in design parameters are not clear and there are several misconceptions. To clarify these effects on sound characteristics and playing conditions, an experiment has been carried out in collaboration with the University of Music and Performing Arts of Vienna (MDW). To eliminate the inconsistency in human playing, an artificial blowing machine (Figure 7) developed in MDW has been used to test the mouthpieces (Chatziioannou et al., 2017). The design parameters tested in the experiment bases on the existing literature and manufacturer definitions (Teal, 1963). The definition of these parameters slightly varies according to the design of each manufacturer. In this case, the Yanagisawa design is taken as reference. The parameters tested in the experiment are tip opening, lay length, chamber shape, chamber size, throat shape, throat size, baffle height, table length and window length (Figure 3). Chamber shape and throat shape are definitions for the cross-sections of the internal cavity of the mouthpiece and vary from square to circular. Each parameter has been tested at three levels. The upper and lower limits are bounded by the measures of existing mouthpieces in the market, as exemplified in Figure 4. Minimum blowing pressure to produce sound, spectral centroid, loudness and pitch interval have been measured for each mouthpiece. The results of the experiment have revealed the effects of the design changes in a quantifiable manner.

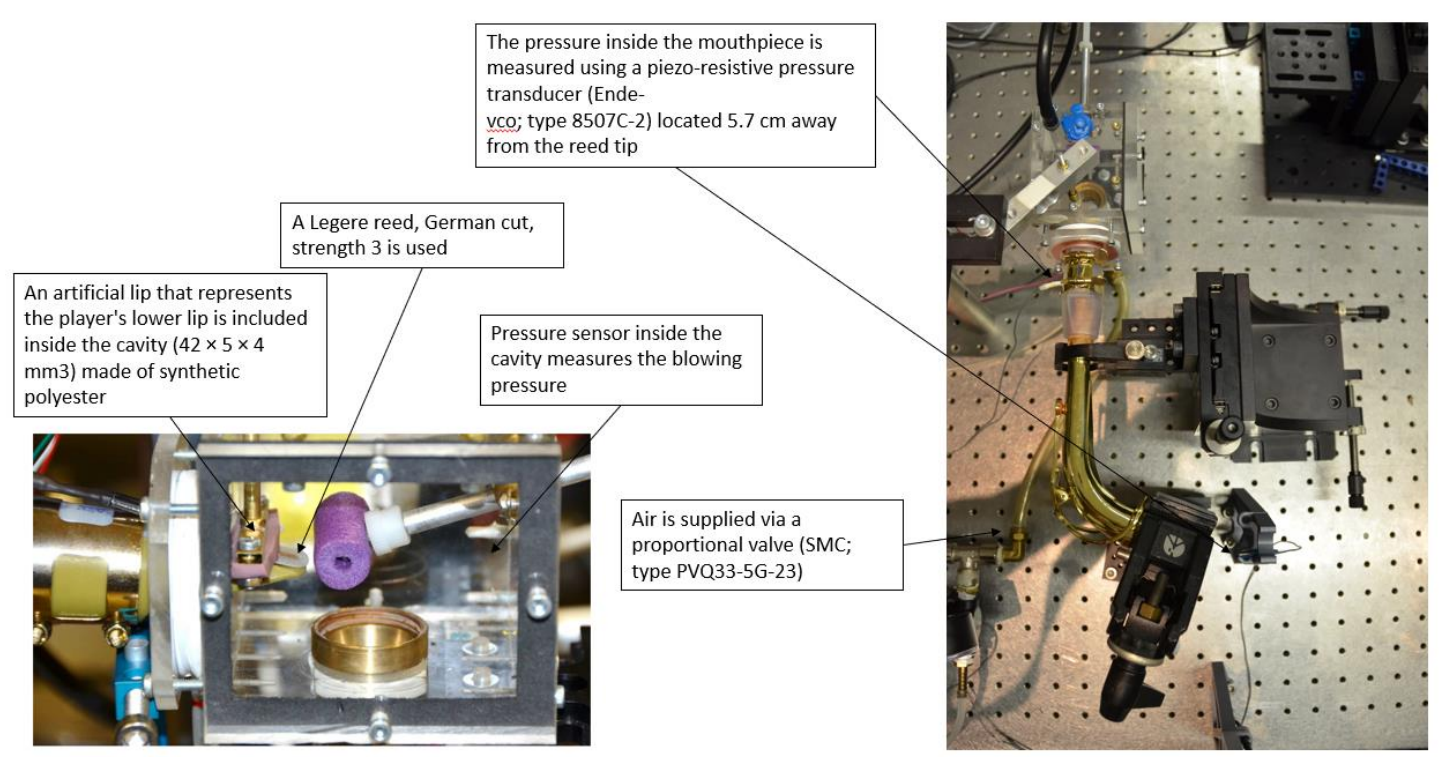

Figure 7. Experiment setup 


\subsection{Example application}

An example of the process is shown in Figure 8 where the user makes the preferences in the order of flexibility, tone colour, resistance and volume. For the sake of clarity, all the FRs and DVs are expressed in a range from 0 to 10 . Since all the variables are free at the beginning, it is possible to choose any value on the scale. Assuming that the user chooses 7 for resistance, to satisfy this requirement, initially TO is set to 0 . Since the limit of TO is reached and the requirement is not met yet, the second variable, $\mathrm{BH}$, is set to 7 and the desired resistance is achieved. Once a selection made, as seen in step 2, the following requirements are restricted due to their dependency on SO or BH. In the second step, the user chooses 3 for tone colour, and this is satisfied by setting TS to 10 and LH to 4 . In the third step, there is only one option left for resistance, since all the variables affecting it has already been set. For the last option, the user chooses 1 for volume, and this is met by setting CS to 10. At this point, all the DVs of the personalized mouthpiece are set, and automatically generated 3D model is ready to be manufactured.
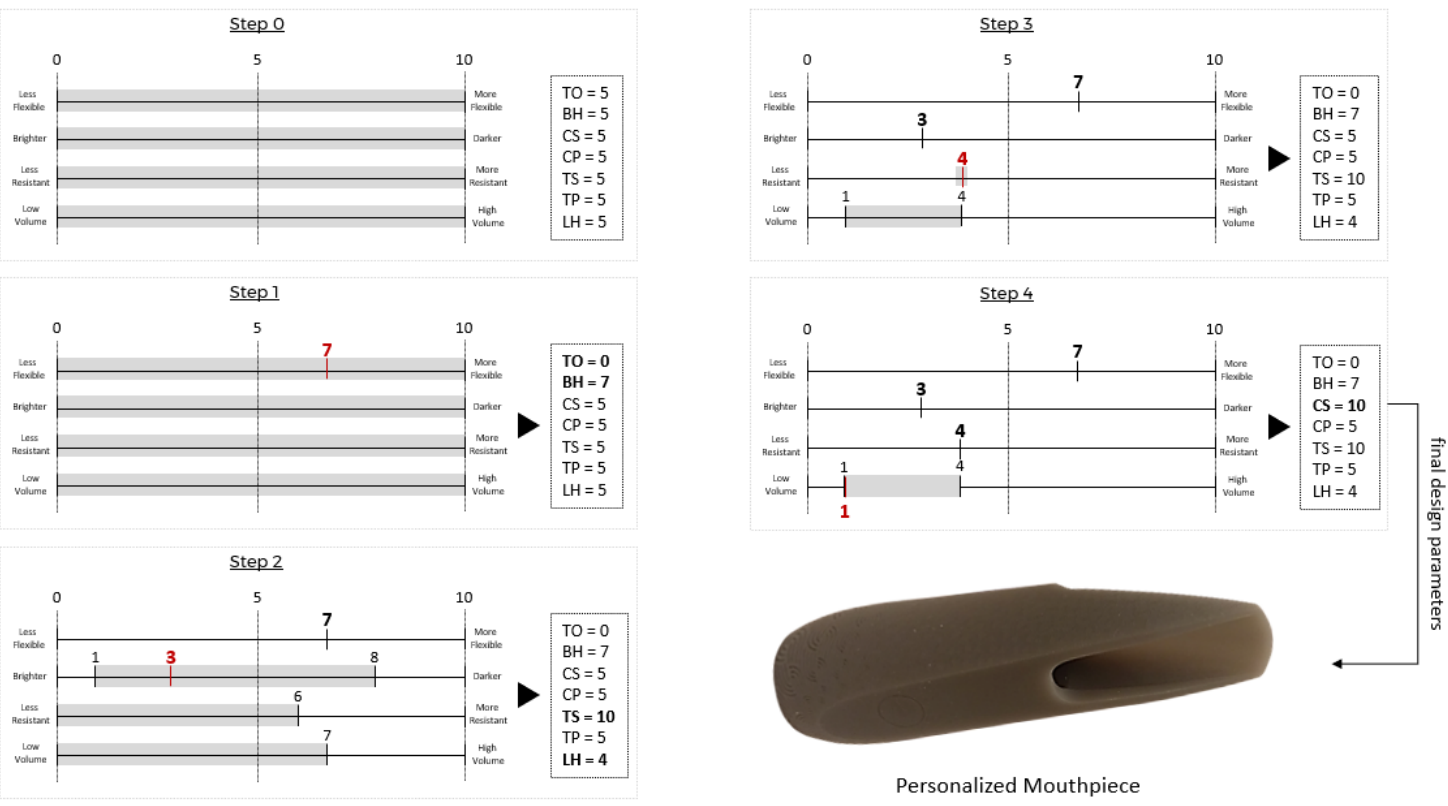

Figure 8. Example application steps

\section{Conclusion and future work}

This case study exemplified a method to develop seed design for the aid of MP product development. How to treat a dynamic product development case with user involvement is described. The work on the saxophone mouthpieces is focused on functional personalization; however, the same concept can be extended to aesthetical and ergonomic personalization cases as well.

The findings of the study reveal that the main pillars of seed design development for MP are the identification of FRs, DVs and deduction of the dynamic design space regarding their dependencies. This requires proper identification of user needs or desires and definition of design parameters to be manipulated to answer these needs. The design space provided to the user is bounded by both user needs and physical limitations. In the mouthpiece example, while manufacturing does not impose significant limitations, desirable features and physical limits of mouthpiece geometry bounds the design space. Therefore, in this case, the design is driven by the already established design parameters, and this defines what is provided to the user.

To demonstrate the applicability of the method in other cases, a brief example on eyewear frames is given. Assumingly, the user is able to decide the weight, colour and the texture of the frame. To answer these, material, topology and surface finish are chosen as design variables. In this case, the weight would be defined by material and topology, while also these are interdependent, and the design space would have a lower boundary by the strength of the frame and upper boundary by user needs. 
By quantifying these dependencies, it is possible to obtain a dynamic design space where user can operate. Similarly, texture and colour would be defined by material and surface finish, which are also interdependent.

The MP of saxophone mouthpieces, whether the concept of automation in answering user needs is applicable, is still under validation. This is planned to be via a user study on saxophone players. The procedure of the validation is briefly composed of co-creation with players and testing of the mouthpieces. Players will be asked to test personalized mouthpieces and some other mouthpieces for reference and provide feedback.

The overall MP process followed in this case study will be verified on a second case study of personalized knitted footwear, which will also include aesthetical and ergonomic aspects of personalization.

\section{References}

Abdul Kudus, S.I., Campbell, R.I. and Bibb, R. (2016), "Assessing the Value of 3D Printed Personalised Products", International Conference on Mass Customization and Personalization in Central Europe, No. November, pp. 1-10.

Ariadi, Y. and Rennie, A.E.W. (2008), "Templates For Consumer Use In Designing Customised Products", Solid Freeform Fabrication Symposium, No. August 2008, pp. 450-458.

Campbell, R.I., Ariadi, Y. and Evans, M.A. (2014), "Facilitating Consumer Involvement in Design", Proceedings of the 5th International Conference on Additive Technologies, No. October 2014, pp. 96-99.

Carron, M. et al. (2017), "Speaking about sounds: a tool for communication on sound features", Journal of Design Research, Vol. 15 No. 2, p. 85.

Chatziioannou, V., Hofmann, A. and Pàmies-Vilà, M. (2017), "An artificial blowing machine to investigate single-reed woodwind instruments under controlled articulation conditions", Vol. 035003 No. 2017, p. 035003.

Christy, D.P. (1993), "Mass Customisation: The New Frontier in Business Competition by B. Joseph Pine II, 1993 (Boston, Harvard Business School Press) US\$29.95, hardback, pp. xxi + 333”, Australian Journal of Management, Vol. 17 No. 2, SAGE PublicationsSage, UK: London, England, pp. 271-283.

Hsiao, W. et al. (2015), "A systematic service design methodology to achieve mass personalisation", International Journal of Agile Systems and Management, Vol. 8 No. 3/4, p. 243.

Kaneko, K. and Kishita, Y. (2017), "In Pursuit of Personalization Design", Procedia CIRP, Elsevier, Vol. 61, pp. 93-97.

Kuksa, I. and Fisher, T. (2017), Design for Personalisation, Design for Personalisation, available at: https://doi.org/10.4324/9781315576633

Laverne, F. et al. (2015), "Assembly Based Methods to Support Product Innovation in Design for Additive Manufacturing: An Exploratory Case Study", Journal of Mechanical Design, American Society of Mechanical Engineers, Vol. 137 No. 12, p. 121701.

Lorenzoni, V., Doubrovski, E.L. and Verlinden, J.C. (2013), "Embracing the digital in instrument making: Towards a musician-tailored mouthpiece by 3D printing", Proceedings of the Stockholm Music Acoustics Conference 2013, SMAC 2013, Stockholm (Sweden), 30 July-3 August, 2013, available at: https://repository.tudelft.nl/ islandora/object/uuid\%3A78ed4e7b-f66e-4ccd-bda0-5eb2243c6b01 (accessed 19 October 2018).

Pahl, G. et al. (2007), "Engineering Design”, A NASA STI/Recon Technical Report. available at: https://doi.org/ $10.1007 / 978-1-84628-319-2$

Rias, A.L. et al. (2016), "Design for additive manufacturing: A creative approach", Proceedings of International Design Conference, Design, Vol. DS 84, pp. 411-420.

Salonitis, K. (2016), "Design for additive manufacturing based on the axiomatic design method", The International Journal of Advanced Manufacturing Technology, Vol. 87 No. 1-4, pp. 989-996.

Sinclair, M. and Campbell, I. (2014), "A Classification of Consumer Involvement in New Product Development", Proceedings of DRS 2014: Design's Big Debates, pp. 1582-1598.

Siu, K.W.M. (2003), “Users' Creative Responses and Designers' Roles”, Design Issues, Vol. 19 No. 2, pp. 64-73.

Spallek, J., Sankowski, O. and Krause, D. (2016), "Influences of additive manufacturing on design processes for customised products", Proceedings of International Design Conference, Design, Vol. DS 84 No. May, pp. 513-522.

Teal, L. (1963), The Art of Saxophone Playing, available at: https://books.google.com/books?id=OeRu0BW V5AgC\&pgis=1

Tseng, M.M., Jiao, R.J. and Wang, C. (2010), "Design for mass personalization", CIRP Annals - Manufacturing Technology, CIRP, Vol. 59 No. 1, pp. 175-178. 
Ulrich, K.T. and Eppinger, S.D. (2012), "Product Design and Development Product Design and Development", available at: https://doi.org/10.1016/B978-0-7506-8985-4.00002-4

Vanessa Rae Hasbrook, B.B. (1996), “Alto saxophone mouthpiece pitch and its relation to jazz and classical tone qualities", available at: http://webpages.uidaho.edu/sax/PDFs/HasbrookThesis.pdf (accessed 11 July 2018).

Wind, J. and Rangaswamy, A. (2001), "Customerization: The next revolution in mass customization", Journal of Interactive Marketing, Vol. 15 No. 1, pp. 13-32.

Zhu, Z. et al. (2017), “A framework for designing end use products for direct manufacturing using additive manufacturing technologies", Proceedings of the International Conference on Engineering Design, ICED, Vol. 5 No. DS87-5, pp. 327-336.

Zipkin, P. (2001), “The Limits of Mass Customization”, MIT Sloan Management Review, Vol. 42 No. 3, pp. 81-87. 